\title{
Forward Kinematics of the General Triple-Arm Robot Using a Distance-Based Formulation
}

\author{
Nicolas Rojas ${ }^{1}$ and Federico Thomas ${ }^{2}$ \\ ${ }^{1}$ Dyson School of Design Engineering, Imperial College, London SW7 1NA, UK \\ e-mail:n.rojas@imperial.ac.uk \\ ${ }^{2}$ Institut de Robòtica i Informàtica Industrial (CSIC-UPC), Barcelona, Spain \\ e-mail: fthomas@iri.upc.edu
}

\begin{abstract}
Distance-based formulations have successfully been used to obtain closure polynomials for planar mechanisms without relying, in most cases, on variable eliminations. The methods resulting from previous attempts to generalize these techniques to spatial mechanisms exhibit some limitations such as the impossibility of incorporating orientation constraints. For the first time, this paper presents a complete satisfactory generalization. As an example, it is applied to obtain a closure polynomial for the the general triple-arm parallel robot (that is, the 3-RPS 3-DOF robot). This polynomial, not linked to any particular reference frame, is obtained without variable eliminations or tangent-half-angle substitutions.
\end{abstract}

Key words: Triple-arm parallel robot, Distance-based formulations, Forward kinematics.

\section{Introduction}

The distance-based formulation introduced in this paper generalizes the ideas developed in [1] for the position analysis of planar kinematic chains to solve the position analysis of spatial mechanisms. The methods resulting from previous attempts to attain this generalization were limited in scope [2], or were unable to obtain closure polynomials of minimum degree for mechanisms with orientation constraints [3]. The proposed formulation permits the incorporation of this kind of constraints so that it can be applied to general spatial linkages. Besides being general, it is shown how it highly simplifies the algebraic manipulations needed to obtain closure polynomials up to the point in which no variable eliminations are needed in many nontrivial cases. As an example, it is applied to obtain a closure polynomial for the the general triple-arm parallel robot (see Fig. 1). This robot consists of a moving platform connected to a fixed base through three revolute-prismatic-spherical kinematic chains, the prismatic joint of each chain being actuated. The forward kinematics problem of this robot consists in finding the possible poses of the moving platform, with respect to the fixed base, for specified values of the actuated prismatic joints. Several researchers have studied this problem (e.g., $[4,5,6,7])$, but their solutions assume that the axes of the revolute joints attached to the fixed base are arranged 


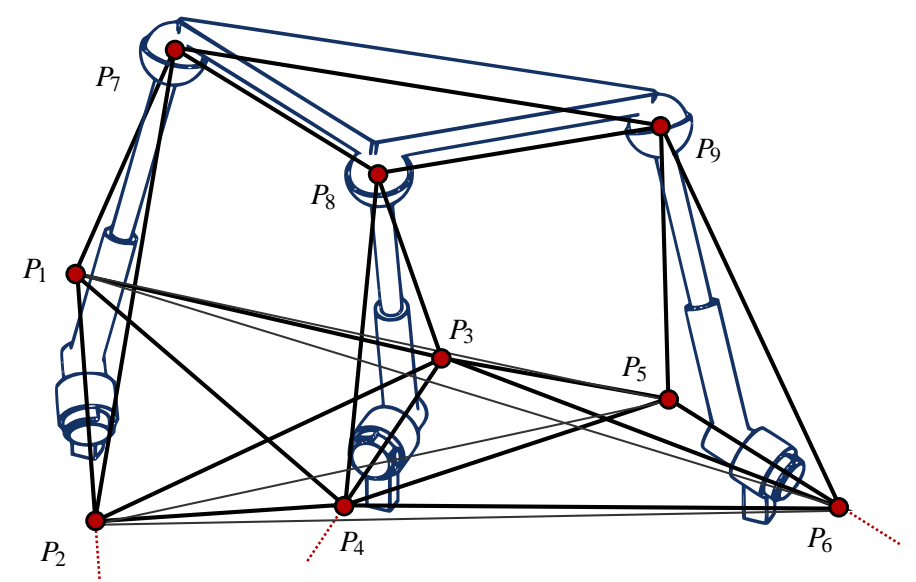

Fig. 1 Triple-arm parallel robot in which the axes supporting the segments $P_{1} P_{2}, P_{3} P_{4}$, and $P_{5} P_{6}$ are skew and its corresponding bar-and-joint framework model.

forming a triangle. In this paper, using the aforementioned distance-based formulation, the forward kinematics of the triple-arm parallel robot with skew revolute joints is solved. It will be shown how this formulation allows obtaining a closure univariate polynomial that is not linked to any particular reference frame, and is straightforwardly obtained without variable eliminations or tangent-half-angle substitutions.

The rest of this paper is organized as follows. Section 2 introduces the basics of the distance-based formulation and its corresponding properties and operations. These ideas are then applied to obtain a closure polynomial for the general triplearm robot in Section 3 which is then applied to solve, in Section 4, its forward kinematics for a particular instance. Finally, we conclude in Section 5.

\section{Preliminaries}

In what follows, $P_{i}$ will denote a point in $\mathbb{E}^{3}, \mathbf{p}_{i, j}=\overrightarrow{P_{i} P_{j}}, \mathbf{p}_{i, j, k}=\mathbf{p}_{i, j} \times \mathbf{p}_{i, k}$, and $s_{i, j}=\left\|\mathbf{p}_{i, j}\right\|^{2}$. Vector coordinates will be arranged as column vectors. Vectors $\mathbf{p}_{i, j}$, $\mathbf{p}_{i, k}$, and $\mathbf{p}_{i, j, k}$ represent, in general, a non-orthonormal reference frame which will be denoted by the column vector of nine components $\mathbf{q}_{i, j, k}=\left(\begin{array}{lll}\mathbf{p}_{i, j}^{T} & \mathbf{p}_{i, k}^{T} & \mathbf{p}_{i, j, k}^{T}\end{array}\right)^{T}$.

The tetrahedron defined by $P_{i}, P_{j}, P_{k}$, and $P_{k}$ will be denoted by $\triangle_{i, j, k, l}$, and it will be said that its origin is located at $P_{i}$, its base is given by then triangle $\triangle_{i, j, k}$, its base vectors are $\mathbf{p}_{i, j}$ (first) and $\mathbf{p}_{i, k}$ (second), and its output vectors are $\mathbf{p}_{i, l}, \mathbf{p}_{j, l}$, and $\mathbf{p}_{k, l}$. 


\subsection{Trilateration in matrix form}

Given the tetrahedron $\triangle_{i, j, k, l}$, its output vector $\mathbf{p}_{i, l}$ can be expressed as a function of the base vectors $\mathbf{p}_{i, j}$ and $\mathbf{p}_{i, k}$ and its squared edge distances as follows (see [8] for details):

$$
a_{i, j, k} \mathbf{p}_{i, l}=b_{i, j, k, l} \mathbf{p}_{i, j}+c_{i, j, k, l} \mathbf{p}_{i, k}+\sigma_{i, j, k, l} d_{i, j, k, l} \mathbf{p}_{i, j, k},
$$

where

$$
\begin{aligned}
& a_{i, j, k}=-\frac{1}{4}\left|\begin{array}{cccc}
0 & 1 & 1 & 1 \\
1 & 0 & s_{i, j} & s_{i, k} \\
1 & s_{i, j} & 0 & s_{j, k} \\
1 & s_{i, k} & s_{j, k} & 0
\end{array}\right|, \quad b_{i, j, k, l}=\frac{1}{4}\left|\begin{array}{cccc}
0 & 1 & 1 & 1 \\
1 & 0 & s_{i, k} & s_{i, l} \\
1 & s_{i, j} & s_{j, k} & s_{j, l} \\
1 & s_{i, k} & 0 & s_{k, l}
\end{array}\right|, \\
& c_{i, j, k, l}=-\frac{1}{4}\left|\begin{array}{cccc}
0 & 1 & 1 & 1 \\
1 & 0 & s_{i, j} & s_{i, l} \\
1 & s_{i, j} & 0 & s_{j, l} \\
1 & s_{i, k} & s_{j, k} & s_{k, l}
\end{array}\right|, \quad d_{i, j, k, l}=\sqrt{\frac{1}{8}\left|\begin{array}{ccccc}
0 & 1 & 1 & 1 & 1 \\
1 & 0 & s_{i, j} & s_{i, k} & s_{i, l} \\
1 & s_{i, j} & 0 & s_{j, k} & s_{j, l} \\
1 & s_{i, k} & s_{j, k} & 0 & s_{k, l} \\
1 & s_{i, l} & s_{j, l} & s_{k, l} & 0
\end{array}\right|,}
\end{aligned}
$$

and $\sigma_{i, j, k, l}$ accounts for the two possible locations of $P_{l}$ with respect to the plane supporting $\triangle_{j, k, l}$ such that $\sigma_{i, j, k, l}=+1$ if $\left|\mathbf{p}_{i, j} \mathbf{p}_{i, k} \mathbf{p}_{i, l}\right|>0$, and $\sigma_{i, j, k, l}=-1$ otherwise.

Equation (1) can be expressed in matrix form as

$$
\mathbf{p}_{i, l}=\mathbf{W}_{i, j, k, l} \mathbf{q}_{i, j, k}
$$

where $\mathbf{W}_{i, j, k, l}=\left(\frac{b_{i, j, k, l}}{a_{i, j, k}} \mathbf{I} \frac{c_{i, j, k, l}}{a_{i, j, k}} \mathbf{I} \frac{d_{i, j, k, l} \mathbf{I}}{a_{i, j, k}}\right), \mathbf{I}$ being the $3 \times 3$ identity matrix. Thus, the output vector $\mathbf{p}_{j, l}$ can be expressed as

$$
\mathbf{p}_{j, l}=\mathbf{p}_{i, l}-\mathbf{p}_{i, j}=\left(\mathbf{W}_{i, j, k, l}-\mathbf{K}_{\mathrm{IOO}}\right) \mathbf{q}_{i, j, k},
$$

where $\mathbf{K}_{\mathrm{IOO}}=\left(\begin{array}{lll}\mathbf{I} & \mathbf{O} & \mathbf{O}\end{array}\right)$, $\mathbf{O}$ being the $3 \times 3$ null matrix. Similarly, for the case of the output vector $\mathbf{p}_{k, l}$ we have that

$$
\mathbf{p}_{k, l}=\mathbf{p}_{i, l}-\mathbf{p}_{i, k}=\left(\mathbf{W}_{i, j, k, l}-\mathbf{K}_{\mathrm{OIO}}\right) \mathbf{q}_{i, j, k},
$$

with $\mathbf{K}_{\mathrm{OIO}}=\left(\begin{array}{lll}\mathbf{O} & \mathbf{I} & \mathbf{O}\end{array}\right)$.

Since $\mathbf{q}_{i, j, k}$ represents, in general, a non-orthogonal reference frame, any vector $\mathbf{v}$ can be expressed as $\mathbf{v}=\boldsymbol{\Omega} \mathbf{q}_{i, j, k}$, where $\boldsymbol{\Omega}=\left(\begin{array}{lll}\omega_{1} \mathbf{I} & \omega_{2} \mathbf{I} & \omega_{3} \mathbf{I}\end{array}\right)$ with $\omega_{i}$ being a scalar. Moreover, it can be checked that $\|\mathbf{v}\|^{2}=\mathbf{q}_{i, j, k}^{T} \boldsymbol{\Omega}^{T} \boldsymbol{\Omega} \mathbf{q}_{i, j, k}$. 


\subsection{Reference frame change}

Let us suppose that $\mathbf{v}$ can be expressed in the reference frame defined by $\mathbf{q}_{l, m, n}$ as $\mathbf{v}=\boldsymbol{\Omega}_{1} \mathbf{q}_{l, m, n}$, where $\boldsymbol{\Omega}_{1}=\left(\omega_{1}^{1} \mathbf{I} \omega_{2}^{1} \mathbf{I} \omega_{3}^{1} \mathbf{I}\right)$. Let us also assume that the base vectors of $\mathbf{q}_{l, m, n}, \mathbf{p}_{l, m}$ and $\mathbf{p}_{l, n}$, can be expressed in the reference frame defined by $\mathbf{q}_{i, j, k}$ as $\mathbf{p}_{l, m}=\boldsymbol{\Omega}_{2} \mathbf{q}_{i, j, k}$ and $\mathbf{p}_{l, n}=\boldsymbol{\Omega}_{3} \mathbf{q}_{i, j, k}$, where $\boldsymbol{\Omega}_{2}=\left(\omega_{1}^{2} \mathbf{I} \omega_{2}^{2} \mathbf{I} \omega_{3}^{2} \mathbf{I}\right)$, and $\boldsymbol{\Omega}_{3}=$ $\left(\omega_{1}^{3} \mathbf{I} \omega_{2}^{3} \mathbf{I} \omega_{3}^{3} \mathbf{I}\right)$, respectively. Then, it is possible to express $\mathbf{v}$ in the reference frame defined by $\mathbf{q}_{i, j, k}$ as:

$$
\mathbf{v}=\boldsymbol{\Omega}_{1} \mathbf{q}_{l, m, n}=\boldsymbol{\Omega}_{1} \boldsymbol{\Lambda}_{i, j, k}^{\boldsymbol{\Omega}_{2} \boldsymbol{\Omega}_{3}} \mathbf{q}_{i, j, k}
$$

where

$$
\boldsymbol{\Lambda}_{i, j, k}^{\boldsymbol{\Omega}_{2} \boldsymbol{\Omega}_{3}}=\left(\begin{array}{ccc}
\omega_{1}^{2} \mathbf{I} & \omega_{2}^{2} \mathbf{I} & \omega_{3}^{2} \mathbf{I} \\
\omega_{1}^{3} \mathbf{I} & \omega_{2}^{3} \mathbf{I} & \omega_{3}^{3} \mathbf{I} \\
k_{1} \mathbf{I} & k_{2} \mathbf{I} & k_{3} \mathbf{I}
\end{array}\right)
$$

with

$$
\begin{aligned}
& k_{1}=\frac{1}{2}\left(\omega_{1}^{2} \omega_{3}^{3}-\omega_{3}^{2} \omega_{1}^{3}\right)\left(s_{i, j}+s_{i, k}-s_{j, k}\right)+\left(\omega_{2}^{2} \omega_{3}^{3}-\omega_{3}^{2} \omega_{2}^{3}\right) s_{i, k}, \\
& k_{2}=-\left(\left(\omega_{1}^{2} \omega_{3}^{3}-\omega_{3}^{2} \omega_{1}^{3}\right) s_{i, j}+\frac{1}{2}\left(\omega_{2}^{2} \omega_{3}^{3}-\omega_{3}^{2} \omega_{2}^{3}\right)\left(s_{i, j}+s_{i, k}-s_{j, k}\right)\right), \\
& k_{3}=\omega_{1}^{2} \omega_{2}^{3}-\omega_{2}^{2} \omega_{1}^{3} .
\end{aligned}
$$

$\boldsymbol{\Lambda}_{i, j, k}^{\boldsymbol{\Omega}_{2} \boldsymbol{\Omega}_{3}}$ is defined as a reference frame change matrix. In the particular case in which $\omega_{1}^{2}=1, \omega_{2}^{2}=\omega_{3}^{2}=0$, this matrix will be explicitly denoted as $\Lambda_{i, j, k}^{\mathbf{K}_{\mathrm{IOO}} \boldsymbol{\Omega}_{3}}$. Likewise, if $\omega_{2}^{3}=1, \omega_{1}^{3}=\omega_{3}^{3}=0$, this matrix will be denoted as $\Lambda_{i, j, k}^{\boldsymbol{\Omega}_{2} \mathbf{K}_{\mathrm{OIO}}}$.

\section{Deriving a closure polynomial for the general triple-arm robot}

A link connecting two skew revolute axes can be modeled by taking two points on each of these axes and connecting them all with edges to form a tetrahedron. Similarly, a link connecting a revolute axis and a ball joint can be modeled by taking two points on the axis and the center of rotation of the spherical pair and connecting them all with edges to form a triangle, and a link connecting two ball joints can be modeled by connecting the centers of rotation of the spherical pairs by an edge. Thus, a triple arm mechanism with skew revolute joints can be modeled as the barand-joint framework shown in Fig. 1. The geometry of this robot is then completely determined by 9 points, namely $P_{1} \ldots P_{9} ; 24$ squared distances, namely $s_{1,2}, s_{1,3}$, $s_{1,4}, s_{1,5}, s_{1,6}, s_{1,7}, s_{2,3}, s_{2,4}, s_{2,5}, s_{2,6}, s_{2,7}, s_{3,4}, s_{3,5}, s_{3,6}, s_{3,8}, s_{4,5}, s_{4,6}, s_{4,8}, s_{5,6}, s_{5,9}$, $s_{6,9}, s_{7,8}, s_{7,9}$, and $s_{8,9}$; and the orientation of 3 tetrahedra, namely $\triangle_{1,2,3,4}, \Delta_{1,2,4,5}$, and $\Delta_{1,2,5,6}$. 
According to the notation of Fig. 1, and applying the operations introduced in Section 2, next we derive a closure condition for this particular robot. To this end, we are going to express $s_{6,9}$ as a function of $s_{3,7}$. In other words, $s_{3,7}$ is going to be used as a parameter in terms of which the configuration of the robot can be expressed.

For the fixed base, which involves points $P_{1} \ldots P_{6}$ and tetrahedra $\Delta_{1,2,3,4}, \Delta_{1,2,4,5}$, and $\Delta_{1,2,5,6}$, we have

$$
\begin{aligned}
& \mathbf{p}_{1,4}=\mathbf{W}_{1,2,3,4} \mathbf{q}_{1,2,3}, \\
& \mathbf{p}_{1,5}=\mathbf{W}_{1,2,4,5} \mathbf{q}_{1,2,4}=\mathbf{W}_{1,2,4,5} \Lambda_{1,2,3}^{\mathbf{K}_{\mathrm{IOO}} \mathbf{W}_{1,2,3,4}} \mathbf{q}_{1,2,3} \\
& \mathbf{p}_{1,6}=\mathbf{W}_{1,2,5,6} \mathbf{q}_{1,2,5}=\mathbf{W}_{1,2,5,6} \Lambda_{1,2,4}^{\mathbf{K}_{\mathrm{IOO}} \mathbf{W}_{1,2,4,5}} \Lambda_{1,2,3}^{\mathbf{K}_{\mathrm{IOO}} \mathbf{W}_{1,2,3,4}} \mathbf{q}_{1,2,3} .
\end{aligned}
$$

Equations (6), (7), and (8) correspond to a representation of the vectors $\mathbf{p}_{1,4}, \mathbf{p}_{1,5}$, and $\mathbf{p}_{1,6}$ in the non-orthonomal reference frame defined by $\mathbf{q}_{1,2,3}$. Now, we derive a representation of $\mathbf{p}_{1,7}$ and $\mathbf{p}_{7,9}$ in the same reference frame to compute the closure vector equation $\mathbf{p}_{6,9}=-\mathbf{p}_{1,6}+\mathbf{p}_{1,7}+\mathbf{p}_{7,9}$. For the case of vector $\mathbf{p}_{1,7}$, we straightforwardly have

$$
\mathbf{p}_{1,7}=\mathbf{W}_{1,2,3,7} \mathbf{q}_{1,2,3} .
$$

For the case of vector $\mathbf{p}_{7,9}$, we first compute

$$
\mathbf{p}_{7,3}=-\mathbf{p}_{3,7}=-\left(\mathbf{W}_{1,2,3,7}-\mathbf{K}_{\mathrm{OIO}}\right) \mathbf{q}_{1,2,3}=\boldsymbol{\Omega}_{73} \mathbf{q}_{1,2,3} .
$$

Similarly, from equations (6) and (9), we get

$$
\mathbf{p}_{7,4}=-\mathbf{p}_{1,7}+\mathbf{p}_{1,4}=\left(\mathbf{W}_{1,2,3,4}-\mathbf{W}_{1,2,3,7}\right) \mathbf{q}_{1,2,3}=\boldsymbol{\Omega}_{74} \mathbf{q}_{1,2,3} .
$$

Then,

$$
\mathbf{p}_{7,8}=\mathbf{W}_{7,3,4,8} \mathbf{q}_{7,3,4}=\mathbf{W}_{7,3,4,8} \boldsymbol{\Lambda}_{1,2,3}^{\boldsymbol{\Omega}_{73} \boldsymbol{\Omega}_{74}} \mathbf{q}_{1,2,3}=\boldsymbol{\Omega}_{78} \mathbf{q}_{1,2,3}
$$

Moreover, from equations (7) and (9), we obtain

$$
\mathbf{p}_{7,5}=-\mathbf{p}_{1,7}+\mathbf{p}_{1,5}=\left(\mathbf{W}_{1,2,4,5} \Lambda_{1,2,3}^{\mathbf{K}_{\mathrm{IOO}} \mathbf{W}_{1,2,3,4}}-\mathbf{W}_{1,2,3,7}\right) \mathbf{q}_{1,2,3}=\boldsymbol{\Omega}_{75} \mathbf{q}_{1,2,3}
$$

and from equations (7), (9), and (12),

$$
\begin{aligned}
\mathbf{p}_{5,8} & =-\mathbf{p}_{1,5}+\mathbf{p}_{1,7}+\mathbf{p}_{7,8} \\
& =\left(-\mathbf{W}_{1,2,4,5} \boldsymbol{\Lambda}_{1,2,3}^{\mathbf{K}_{\mathrm{IOO}} \mathbf{W}_{1,2,3,4}}+\boldsymbol{\Omega}_{78}+\mathbf{W}_{1,2,3,7}\right) \mathbf{q}_{1,2,3}=\boldsymbol{\Omega}_{58} \mathbf{q}_{1,2,3} .
\end{aligned}
$$

Then, using equations (12) and (13), we have

$$
\mathbf{p}_{7,9}=\mathbf{W}_{7,8,5,9} \mathbf{q}_{7,8,5}=\mathbf{W}_{7,8,5,9} \Lambda_{1,2,3}^{\boldsymbol{\Omega}_{78} \boldsymbol{\Omega}_{75}} \mathbf{q}_{1,2,3} .
$$


Using equations (8), (9), and (15), we can now write $\mathbf{p}_{6,9}$ in the reference frame defined by $\mathbf{q}_{1,2,3}$ as:

$$
\mathbf{p}_{6,9}=-\mathbf{p}_{1,6}+\mathbf{p}_{1,7}+\mathbf{p}_{7,9}=\boldsymbol{\Omega}_{69} \mathbf{q}_{1,2,3},
$$

with

$$
\boldsymbol{\Omega}_{69}=-\mathbf{W}_{1,2,5,6} \Lambda_{1,2,4}^{\mathbf{K}_{\mathrm{IOO}} \mathbf{W}_{1,2,4,5}} \Lambda_{1,2,3}^{\mathbf{K}_{\mathrm{IOO}} \mathbf{W}_{1,2,3,4}}+\mathbf{W}_{7,8,5,9} \boldsymbol{\Lambda}_{1,2,3}^{\boldsymbol{\Omega}_{78} \boldsymbol{\Omega}_{75}}+\mathbf{W}_{1,2,3,7} .
$$

Thus, we finally conclude that

$$
s_{6,9}=\mathbf{q}_{1,2,3}^{T} \boldsymbol{\Omega}_{69}^{T} \boldsymbol{\Omega}_{69} \mathbf{q}_{1,2,3} .
$$

The right hand side of the above equation is a function of the unknown squared distances $s_{3,7}, s_{4,7}, s_{5,7}$ and $s_{5,8}$. However, from equations (11), (13), and (14), we have that

$$
\begin{aligned}
& s_{4,7}=\mathbf{q}_{1,2,3}^{T} \boldsymbol{\Omega}_{74}^{T} \boldsymbol{\Omega}_{74} \mathbf{q}_{1,2,3}, \\
& s_{5,7}=\mathbf{q}_{1,2,3}^{T} \boldsymbol{\Omega}_{75}^{T} \boldsymbol{\Omega}_{75} \mathbf{q}_{1,2,3}, \\
& s_{5,8}=\mathbf{q}_{1,2,3}^{T} \boldsymbol{\Omega}_{58}^{T} \boldsymbol{\Omega}_{58} \mathbf{q}_{1,2,3} .
\end{aligned}
$$

Then, the substitution of these expressions in (17) yields a scalar radical equation in a single variable: $s_{3,7}$. The real roots of this closure condition determine the assembly modes of the analyzed robot. These roots can be computed, for instance, from the univariate polynomial resulting from clearing the radicals in this expression, as explained in [9]. For each real root, we can determine the location of the three points of the moving platform by computing, for example, the following sequence of trilaterations: obtaining $\mathbf{p}_{1,7}$ from $\mathbf{p}_{1,2}$ and $\mathbf{p}_{1,3}$, then $\mathbf{p}_{3,8}$ from $\mathbf{p}_{3,7}$ and $\mathbf{p}_{3,4}$, and finally $\mathbf{p}_{5,9}$ from $\mathbf{p}_{5,7}$ and $\mathbf{p}_{5,8}$. This leads to up to eight locations for $P_{9}$. At least one of them necessarily satisfies the distance constraint imposed by $s_{6,9}$ and hence corresponds to a valid assembly mode.

\section{Numerical example}

According to the notation of Fig. 1, let us consider the triple arm mechanism with the following known squared lengths: $s_{1,2}=1, s_{1,3}=17, s_{1,4}=10, s_{1,5}=26, s_{1,6}=20$, $s_{1,7}=101, s_{2,3}=16, s_{2,4}=11, s_{2,5}=19, s_{2,6}=13, s_{2,7}=102, s_{3,4}=3, s_{3,5}=11$, $s_{3,6}=13, s_{3,8}=126, s_{4,5}=20, s_{4,6}=18, s_{4,8}=101, s_{5,6}=2, s_{5,9}=145, s_{6,9}=123$, $s_{7,8}=10, s_{7,9}=26$, and $s_{8,9}=10$; with $\sigma_{1,2,3,4}=+1, \sigma_{1,2,4,5}=-1$, and $\sigma_{1,2,5,6}=$ +1 . Substituting these values in (17), using the expressions for $s_{4,7}, s_{5,7}$ and $s_{5,8}$ in terms of $s_{3,7}$, and clearing radicals, we obtain the following polynomial: 


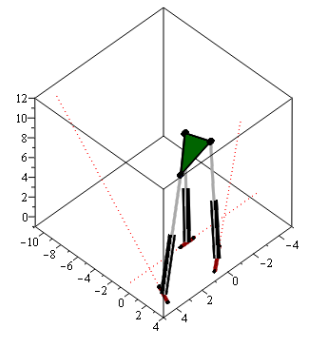

$s_{3,7}=126.00$

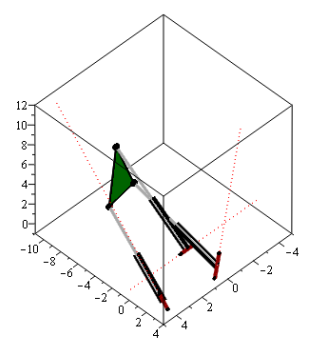

$s_{3,7}=190.26$

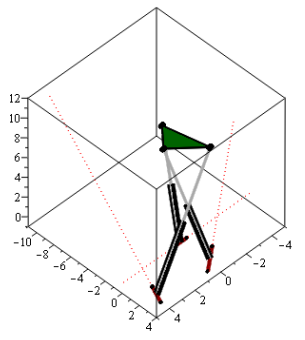

$s_{3,7}=140.93$

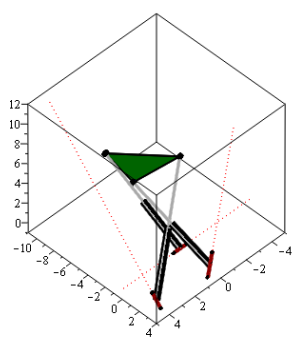

$s_{3,7}=193.73$

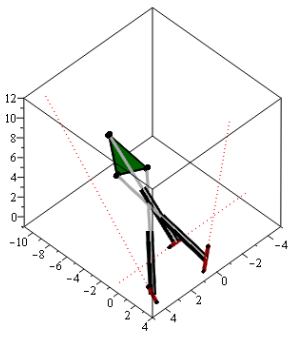

$s_{3,7}=186.75$

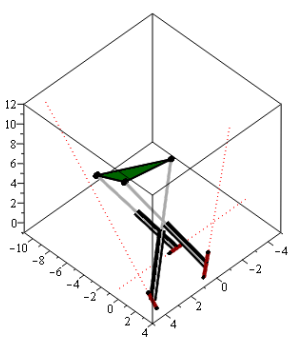

$s_{3,7}=198.13$

Fig. 2 The six real solutions to the forward kinematics of the analyzed triple-arm robot.

$$
\begin{aligned}
& 4.3635 \cdot 10^{12} s_{3,7}^{16}-1.1184 \cdot 10^{16} s_{3,7}^{15}+1.3683 \cdot 10^{19} s_{3,7}^{14}-1.0517 \cdot 10^{22} s_{3,7}^{13} \\
& +5.6413 \cdot 10^{24} s_{3,7}^{12}-2.2259 \cdot 10^{27} s_{3,7}^{11}+6.6546 \cdot 10^{29} s_{3,7}^{10}-1.5332 \cdot 10^{3} 2 \cdot s_{3,7}^{9} \\
& +2.7456 \cdot 10^{3} 4 \cdot s_{3,7}^{8}-3.8296 \cdot 10^{3} 6 \cdot s_{3,7}^{7}+4.1433 \cdot 10^{3} 8 \cdot s_{3,7}^{6}-3.4390 \cdot 10^{40} s_{3,7}^{5} \\
& +2.1463 \cdot 10^{42} s_{3,7}^{4}-9.7358 \cdot 10^{43} s_{3,7}^{3}+3.0275 \cdot 10^{45} s_{3,7}^{2}-5.7676 \cdot 10^{46} s_{3,7} \\
& +5.0725 \cdot 10^{47} .
\end{aligned}
$$

This polynomial has six real roots: $126.00,140.93,186.75,190.26,193.73$, and 198.13. The corresponding robot configurations for the case in which $P_{1}$ is located at the origin, and $\mathbf{p}_{1,2}=(1,0,0)^{T}, \mathbf{p}_{1,3}=(1,4,0)^{T}, \mathbf{p}_{1,4}=(0,3,1)^{T}, \mathbf{p}_{1,5}=$ $(4,3,-1)^{T}$, and $\mathbf{p}_{1,6}=(4,2,0)^{T}$, appear in Fig. 2 .

\section{Conclusion}

Solving the position analysis of kinematic chains based on the idea of obtaining closure conditions using n-laterations and constructive geometry arguments has been quite successful for the planar case. However, the extension of this approach to three dimensions, to solve the position analysis of spatial mechanisms, remained elusive 
despite the efforts to generalize the planar techniques. The main drawbacks of these previous attempts include the impossibility of dealing with orientation constraints, the limited range of mechanisms that can be analyzed using them, and the complexity of the algebraic manipulation needed to solve even relatively simple problems. This paper has introduced the basic concepts and properties of a distance-based matrix formulation that clears all these disadvantages. The technique has been applied to solve the forward kinematics of the triple arm mechanism with skew revolute joints.

Acknowledgements This work has been partially supported by the Spanish Ministry of Economy and Competitiveness under project DPI2014-57220-C2-2-P.

\section{References}

1. N. Rojas, "Distance-based formulations for the position analysis of kinematic chains," Ph.D. thesis, Universitat Politècnica de Catalunya, 2012

2. N. Rojas and F. Thomas, "The closure condition of the double banana and its application to robot position analysis," in Proceedings of the IEEE International Conference on Robotics and Automation, 2013, pp. 46414646

3. F. Thomas and J.M. Porta, "Closure polynomials for strips of tetrahedra," in Advances in Robot Kinematics, 2016

4. C. Gosselin, "Kinematic analysis optimization and programming of parallel robotic manipulators," Ph.D. Thesis, McGill University, 1988

5. K.J. Waldron, M. Raghavan, and B. Roth, "Kinematics of a hybrid series-parallel manipulation system," ASME Journal of Mechanisms, Transmissions, and Automation in Design, vol. 111, no. 2, pp. 211-221, 1989

6. J. Kim and F.C. Park, "Direct kinematic analysis of 3-RS parallel mechanisms," Mechanism and Machine Theory, vol. 36, no. 10, pp. 1121-1134, 2001

7. J. Schadlbauer, D. Walter, and M. Husty, “A complete analysis of the 3-RPS manipulator,' In: G. Bandyopadhyay and P. Ramu (eds), Machines and Mechanisms, Narosa Publishing House, pp. 410-419, 2011.

8. F. Thomas and L. Ros, "Revisiting trilateration for robot localization," IEEE Transactions on Robotics, vol. 21, no. 1, pp. 93-101, 2005

9. J.M. Porta and F. Thomas, "Closed-form position analysis of variable geometry trusses," Mechanism and Machine Theory, to appear. 